\title{
COMPARISON OF FLEXIBLE URETERORENOSCOPE QUALITY OF VISION: AN IN VITRO STUDY
}

\author{
Michele Talso ${ }^{1,2,7} \dagger$, Silvia Proietti ${ }^{1,3,7} \dagger$, Esteban Emiliani ${ }^{1,4,7}$, Andrea Gallioli ${ }^{2}$, Laurian
} Dragos $^{1,5,7}$, Andrea Orosa ${ }^{1}$, Pol Servian ${ }^{1}$, Aaron Barreiro ${ }^{1}$, Guido Giusti ${ }^{3}$, Emanuele Montanari ${ }^{2}$, Bhaskar Somani ${ }^{6,7}$, Olivier Traxer ${ }^{1,7}$

\section{On Behalf of the PETRA Group}

1 Sorbonne Université, GRC n²0 LITHIASE RENALE, AP-HP, Hôpital Tenon, F-75020 PARIS, France

2 Department of Urology Fondazione IRCCS Ca' Granda - Ospedale Maggiore Policlinico Università degli Studi di Milano, Italy

3 Department of Urology, San Raffaele Hospital , Ville Turro Division, Milan, Italy

4: Department of Urology Fundación Puigvert, , Universidad Autónoma de Barcelona, Spain

5 Department of Urology University of Medicine and Pharmacy Victor Babes, Timisoara, Romania

6 Department of Urology, University Hospital Southampton NHS Trust, Southampton, UK.

7 Members of PETRA UroGroup, Progress in Endourology, Technology and Research

Association http://www.petraurogroup.org/

† Both authors contribute equally to this study 


\section{INTRODUCTION}

Flexible ureterorenoscopy (fURS) is one of the best solutions for treatment of renal calculi less than $2 \mathrm{~cm}$ and for conservative treatment of upper tract urothelial carcinoma (UTUC) . $^{\mathrm{i}}$ Adequate quality of vision is mandatory if surgeons are to achieve better outcomes. In recent decades, different companies have developed various flexible ureterorenoscope (FU) models. Flexible scopes offer improved vision based on the incorporation of an increased number of fiber optic bundles or the integration of a distal sensor chip ${ }^{\text {ii,iii }}$. Beside multiple-use FUs, seven disposable devices have been developed to date ${ }^{\text {iv }}$. In terms of size, shape, manipulation, and view, the disposable FU most closely comparable to the commonly used multiple-use FUs remains the LithoVue from Boston Scientific ${ }^{4, v}$. To our knowledge, no studies have been done on which FU on the market offers the best quality of vision. We therefore decided to compare the quality of vision provided by the most frequently used digital (D) and fiber optic (FO) FUs.

\section{MATERIALS AND METHODS}

Seven different FUs [P6 (FO) (Olympus ${ }^{\circledR}$ ); V (D) (Olympus ${ }^{\circledR}$ ); V2 (D) (Olympus ${ }^{\circledR}$ ); Flex XC (D) $\left(\right.$ Storz $\left.^{\circledR}\right)$; Flex X2 (FO) (Storz $\left.{ }^{\circledR}\right)$; Cobra Vision (D) (Wolf ${ }^{\circledR}$ ); LithoVue (D) (Boston Scientific ${ }^{\circledR}$ )] were used to record eight different standardized videos of $3 \mathrm{~s}$ each. The Storz Flex XC was used with white light (WL) vision and using the Clara+Chroma modality (CC), which has been reported to be the light mode offering best image quality in the Spies ${ }^{\mathrm{TM}}$ system $^{\mathrm{vi}}$. Apart from the LithoVue, the FUs were not brand new. The selection of these FUs was made on purpose in order to simulate daily practice, where surgeons usually do not employ brand new scopes for fURS. All these scopes were assessed before the study and checked to be functioning and operating properly according to hospital standards. Two standardized grids (Edmund Optics, Barrington, $\mathrm{NJ}^{\circledR}$ ) vii,viii and three stones of different composition (monohydrate calcium oxalate, dehydrate calcium oxalate and uric acid) were used to evaluate color contrast and definition of the images. All videos were taken at $3 \mathrm{~cm}$ from the FC camera. Images of the grids and stones were acquired in four standardized scenarios using the K-Box (Coloplast ${ }^{\circledR}$ ) simulation model. The mediums used were: $110 \mathrm{ml}$ of saline solution, $110 \mathrm{ml}$ of sterile water, $110 \mathrm{ml}$ of saline solution mixed with $3 \mathrm{ml}$ of 
iodine solution $0.3 \%$ (Betadine $^{\circledR}$ ) to simulate bleeding, and $80 \mathrm{ml}$ of saline solution mixed with $30 \mathrm{ml}$ of contrast. Betadine was used since it was not possible to use real human blood to simulate bleeding. In fact, multiple use FUs used for the study were the same used on daily practice on patients. Therefore, use real blood on a bench model was not acceptable by our ethical committee. In total 96 videos were made. Twelve slides, each containing eight videos of the same scenario, were acquired to compare simultaneously all the devices. The position of the different videos was changed randomly in each slide. The angles of the squared digital images were cut in order to give the same circular shape to all videos (Figure 1). These videos were shown to 103 subjects (51 urologists and 52 nonurologists), of whom 33 were women and 70 men, with a mean age of 36 years (range 2559). Each slide containing 8 videos was shown for 15 seconds to the audience. Each 3 seconds single video inside the slide was played in loop. The subjects evaluated each video on a scale from 1 (very bad) to 5 (very good). The total score for every FU was calculated as the mean of each scenario.

Statistical analysis was performed with GraphPad Prism version 5 (GraphPad Software Inc., California, USA). T-tests were used for comparison between two groups. One-way analysis of variance (ANOVA) and Tukey's test were deployed for multiple comparisons. Statistically significant differences were assumed for $p$ values of less than 0.05 . Adequacy of the sample size for comparison of groups was calculated by testing the first 30 cases with G*Power v. 3.1.9.2 ( $\alpha$ 0.05, Power 0.95). The minimum sample size required proved to be 24 .

\section{RESULTS}

No differences in scores were observed according to the sex of the participants. In general, urologists gave significantly higher scores than non-urologists (mean \pm SD $2.84 \pm 0.31$ vs $2.7 \pm 0.33, p=0.04)$. Olympus V/V2 and Cobra Vision had significantly lower mean values in the non-urologist group $(p<0.04)$ while the other FUs had comparable scores. Optical scopes were scored lower than digital ones $(1.3 \pm 0.4$ vs $3.26 \pm 0.42$, respectively, $p<0.0001)$. As shown in Table 1, Flex $X C_{W L}$ and $X C_{C C}$ image quality was consistently better than that of the other FUs $(p<0.0001)$. Olympus $V$ generally provided better vision than LithoVue 
$(3.35 \pm 0.65$ vs $2.76 \pm 0.54$, respectively, $p<0.0001)$. Cobra Vision and Olympus V2 had superimposable values which were significantly lower than those for LithoVue. Olympus P6 and Storz X2 offered a low quality of vision compared with the other FUs.

Consideration of only the images of number grids yielded the same results. Upon consideration of the images of color grids, the LithoVue score was comparable to that of Olympus $V(3.26 \pm 0.63$ vs $3.29 \pm 0.78, p=0.75)$ and both scored significantly higher than Olympus V2 and Cobra Vision ( $p<0.0001)$. On the other hand, for stone images Olympus V2 $(2.69 \pm 0.59)$ received better scores than LithoVue $(2.36 \pm 0.67)$ and Cobra Vision $(2.43 \pm 0.58)$ $(p<0.0001)$ (Figure 2). The results of FUs in saline solution and sterile water resembled the total scores, even if in the former medium the difference between LithoVue and Olympus $V$ was not significant $(2.94 \pm 0.64$ and $3.08 \pm 0.66$, respectively; $p=0.12)$. In contrast-medium solution, Olympus $V(3.57 \pm 0.78)$ provided a good quality of image, far better than Cobra Vision, LithoVue, and Olympus V2 $(2.63 \pm 0.71,2.66 \pm 0.74$, and $2.6 \pm 0.65$, respectively; $p<0.0001$ ), for which the values did not differ significantly (all $p>0.5$ ). In Betadine solution, the Flex $\mathrm{XC}_{\mathrm{cc}}$ still had the best scores but Olympus $\mathrm{V}$ provided an image definition similar to Flex $X C_{W L}(3.45 \pm 0.89$ vs $3.67 \pm 0.78$, respectively, $p=0.07)$. The scores for Cobra Vision $(2.34 \pm 0.64)$ were lower than those for LithoVue and Olympus V2 $(2.58 \pm 0.68$ and $2.62 \pm 0.74$ respectively; both $\mathrm{p}<0.01$ ) (Figure $3 \mathrm{~A}$ ).

To evaluate the difference in image quality between saline and Betadine solution for all FUs, we calculated the difference in scores in the two mediums and analyzed the groups (Table 2). Olympus P6, Storz X2, and Cobra Vision provided a comparable resolution in the two mediums. The image quality scores for Storz $X C_{W L}$ and $X C_{C C}$ were, respectively, $15.9 \%$ and $12.4 \%$ lower in the Betadine solution, and a lower image quality in this medium was also observed for LithoVue (12\%). By contrast, scores for Olympus V and V2 improved significantly in Betadine solution, by $12 \%$ and $8.1 \%$, respectively (Table 3, Figure 3B).

\section{DISCUSSION}

Nowadays, fURS is widely performed. The introduction of digital ureteroscopy has offered diverse advantages for diagnostic and treatment procedures, with a significant reduction in operative times when treating stones ${ }^{\mathrm{ix}}$. Due to the cost of instruments, not all urological centers own both digital and fiber optic FUs. In an era in which there is a free market and it 
is possible to choose between brands, it is useful to know the characteristics of instruments. To our knowledge, however, no studies have yet been done on the comparative image quality delivered by different FUs. It can be difficult for surgeons to decide what instrument to buy for their unit. Moreover, more than one company offers more than one FO and D model. While there is not a specific indication for use of a D or FO scope during stone treatment, D scopes seem to be the right choice in the case of UTUC. In fact, a D scope is the most valuable instrument for evaluation of the intrarenal collecting system and ureter under direct vision in order to allow complete tumor ablation ${ }^{x}$. New D disposable scopes relevant to this scenario are coming onto the market. The most similar to reusable scopes in terms of shape, images, and manipulation is the LithoVue (Boston Scientific) ${ }^{5, x i}$.

In our study, as demonstrated previously ${ }^{\mathrm{xii}}, \mathrm{D}$ scopes resulted in better evaluation than FO scopes. Image quality is a characteristic that does not strictly need to be evaluated only by medical doctors. For this reason we decided to show images also to non-urologists, who seemed to prefer the Olympus V and V2 and Wolf Cobra Vision to the other FUs. The FU that had the best evaluation overall, with a statistically significant difference, was the D scope from Storz (Flex XC) in the white light and Clara+Chroma modalities, where Clara+Chroma had the best rate, as previously demonstrated ${ }^{6}$. The first-generation FU from Olympus (V) seems to offer better vision quality than the newer Olympus V2, probably owing to its larger dimensions. Overall, LithoVue had a better evaluation than Wolf CobraVision and Olympus V2, apparently gaining points with color grids and losing quality on stone images. The latter were well evaluated using the Olympus V2. No differences were found when images were recorded in saline and sterile water, while evaluations using contrast-medium solution revealed that Storz Flex $\mathrm{XC}_{\mathrm{cc}}$ remained the scope with the highest evaluation, followed by Storz $X C_{W L}$ and Olympus V. With both Olympus D scopes, image quality improved significantly in Betadine solution, although worsening of image quality was observed for the remaining D scopes. No significant difference in quality was seen for FO scopes in saline and Betadine solutions. We did not analyze Olympus scopes in the narrow band imaging modality because this modality is specifically indicated for detection of urothelial tumors, whereas Spies ${ }^{\mathrm{TM}}$ modalities are not recommended by the company for any specific situation ${ }^{\text {xiii }}$. Certainly, vision is not the only 
parameter we have to consider while choosing what scope should be used for a surgery. Also diameter and manoeuvrability should be taken in count. Dragos et al. demonstrated that D FUs are less effective in accessing the sharp angled calix and they have lesser endtip deflection compared with the fiber-optic counterparts ${ }^{\text {xiv }}$.

The strengths of this study is the size of the group who evaluated the videos and the fact that this is the first study to compare the image quality offered by the most frequently used FUs on the market. Limitations are the in vitro setting and the impossibility of using real human blood for the experiment, since we used scopes intended for application in daily practice. Finally, not all the scopes available on the market were tested. This preliminary study is not intended to promote one scope over another; rather, it provides information for further in vivo assessments. All scopes analyzed are used commonly in many centers, and each of them has its own pros and cons. The aim of the study was to provide a general idea of the image quality. Beside image quality, manoeuvrability, scope body mass index ${ }^{\mathrm{xv}}$ and costs have to take in consideration before buying a FU.

\section{CONCLUSION}

In this in vitro study, Storz Flex $\mathrm{XC}_{\mathrm{CC}}$ was ranked as the best $\mathrm{FU}$ in terms of image quality. Olympus V was evaluated to be better than V2. Overall, Boston Scientific LithoVue resulted having a better evaluation than Olympus V2 and Wolf Cobra Vision that were comparable. Digital scopes had better evaluations than fiber optic ones.

\section{Disclosure:}

This study was not funded by any company.

At the time of the study,

Professor Olivier Traxer was a consultant for Boston Scientific, Coloplast, Olympus and Rocamed

Dr Guido Giusti was a consultant for Boston Scientific, Coloplast, Cook, Lumenis, Olympus and Rocamed 
Page 7 of 16

7

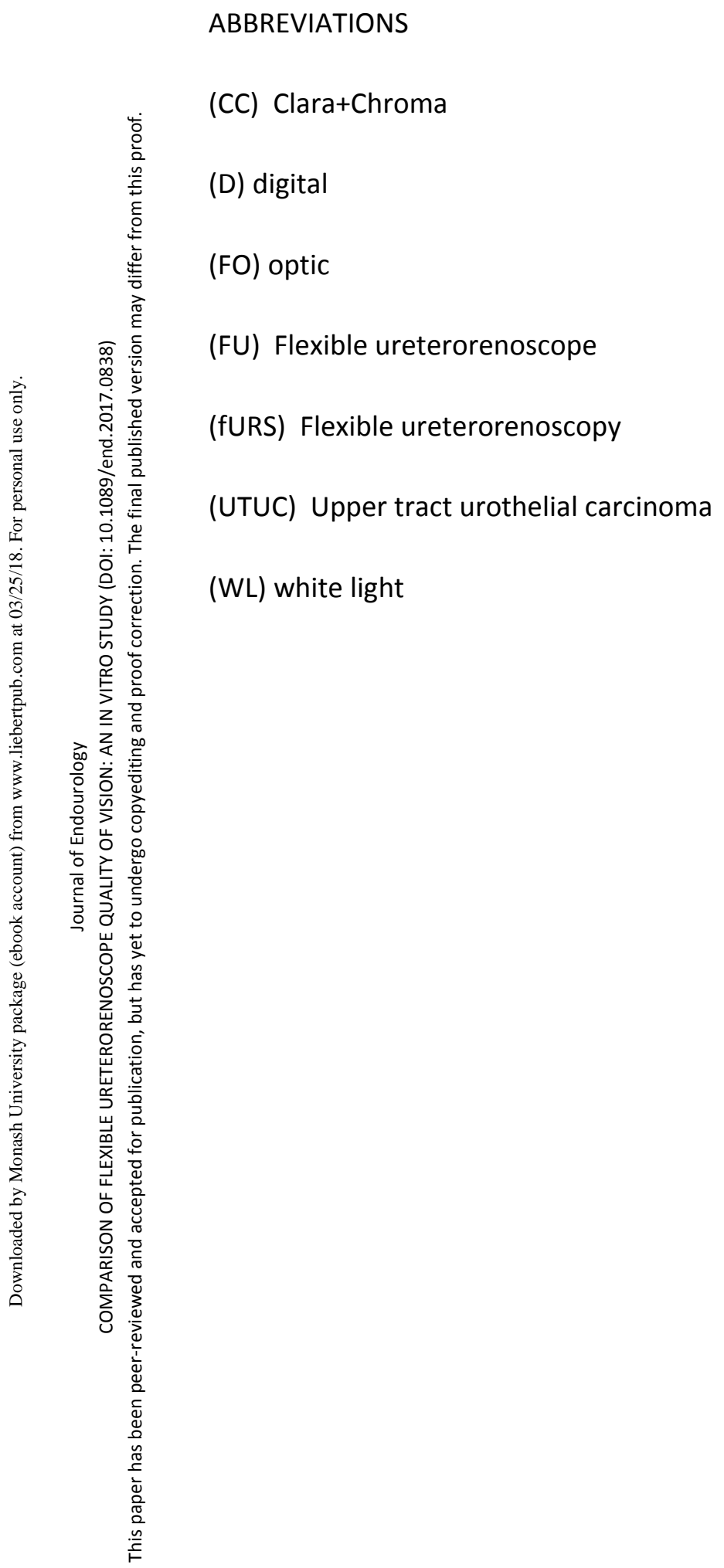


TABLE 1

Mean (standard deviation) fURS scores divided for type of image and medium. Range of score were 1 (very bad) to 5 (very good). Storz digital scopes were evaluated in White Light (WL) and Clara Chroma (CC) mode.

\begin{tabular}{|lcccccccc|} 
& Cobra Vision & Lithovue & Olympus P6 & Olympus V & Olympus V2 & Storz X2 & Storz XC wLwL & Storz XC cccc \\
\hline NaCl & $2,37(0,58)$ & $2,9(0,64)$ & $1,23(0,41)$ & $3,08(0,66)$ & $2,42(0,59)$ & $1,4(0,53)$ & $4,36(0,63)$ & $4,47(0,6)$ \\
\hline Sterile Water & $2,45(0,58)$ & $2,85(0,59)$ & $1,17(0,44)$ & $3,29(0,73)$ & $2,44(0,6)$ & $1,27(0,48)$ & $4,26(0,56)$ & $4,55(0,65)$ \\
\hline Contrast & $2,63(0,71)$ & $2,66(0,74)$ & $1,3(0,56)$ & $3,57(0,78)$ & $2,6(0,65)$ & $1,39(0,5)$ & $4,2(0,69)$ & $4,47(0,72)$ \\
\hline Betadine & $2,34(0,64)$ & $2,58(0,68)$ & $1,23(0,45)$ & $3,45(0,89)$ & $2,62(0,74)$ & $1,44(0,47)$ & $3,67(0,78)$ & $3,92(0,9)$ \\
\hline Numbers & $2,39(0,63)$ & $2,67(0,66)$ & $1,08(0,38)$ & $3,36(0,71)$ & $2,28(0,63)$ & $1,23(0,47)$ & $4,17(0,75)$ & $4,28(0,76)$ \\
\hline Colours & $2,52(0,63)$ & $3,26(0,63)$ & $1,34(0,57)$ & $3,29(0,78)$ & $2,61(0,63)$ & $1,46(0,56)$ & $4,1(0,56)$ & $4,37(0,71)$ \\
\hline Stone & $2,43(0,58)$ & $2,36(0,67)$ & $1,28(0,48)$ & $3,39(0,73)$ & $2,67(0,59)$ & $1,43(0,41)$ & $4,09(0,59)$ & $4,4(0,67)$ \\
\hline Total & $2,45(0,53)$ & $2,76(0,54)$ & $1,23(0,41)$ & $3,35(0,65)$ & $2,52(0,54)$ & $1,37(0,41)$ & $4,12(0,54)$ & $4,35(0,63)$ \\
\hline
\end{tabular}




\section{TABLE 2}

Difference between saline and Betadine solutions scores. To objectify the difference of image quality between saline and iodine solution for all fURS it was calculated the difference of scores in the two mediums and analyzed the groups. Where the difference is negative, it means that iodine solution was better evaluated.

\begin{tabular}{|lccc|}
\hline Model & Mean Saline - Mean & & \\
\hline Cobra Vision & Betadine & Percentage & p value \\
\hline Lithovue & 0.02913 & $-1.2 \%$ & 0.73 \\
\hline Olympus P6 & 0.3592 & $-12.2 \%$ & 0.0001 \\
\hline Olympus V & 0.006472 & $-0.5 \%$ & 0.9 \\
\hline Olympus V2 & -0.3689 & $12 \%$ & 0.0009 \\
\hline Storz X2 & -0.1974 & $8.1 \%$ & 0.04 \\
\hline Storz XC $\mathrm{WL}$ & -0.04531 & $3.3 \%$ & 0.52 \\
\hline Storz XC & 0.6926 & $-15.9 \%$ & $<0,0001$ \\
\hline
\end{tabular}


Table 3

Mean of evaluation of different fURS while recording different targets (first table from the top) and in different medium (second table from the top). Storz XC was evaluated in White Light (WL) and in Clara Chroma (CC) modality.

\begin{tabular}{|c|c|c|c|c|c|c|c|c|}
\hline TARGET & Cobra Vision & Lithovue & Olympus P6 & Olympus V & Olympus V2 & Storz X2 & Storz XC $\mathrm{WLWL}_{\mathrm{W}}$ & Storz XC $\mathrm{Cccc}_{\mathrm{ccc}}$ \\
\hline Numbers & 2.391 & 2.665 & 1.075 & 3.362 & 2.279 & 1.233 & 4.167 & 4.284 \\
\hline Colours & 2.527 & 3.257 & 1.340 & 3.289 & 2.609 & 1.461 & 4.102 & 4.369 \\
\hline Stone & 2.430 & 2.359 & 1.282 & 3.393 & 2.670 & 1.427 & 4.090 & 4.398 \\
\hline Total & 2.449 & 2.761 & 1.232 & 3.348 & 2.519 & 1.374 & 4.120 & 4.350 \\
\hline
\end{tabular}

\begin{tabular}{|c|c|c|c|c|c|c|c|c|}
\hline MEDIUM & Cobra Vision & Lithovue & Olympus P6 & Olympus V & Olympus V2 & Storz X2 & Storz $\mathrm{XC}_{\mathrm{WLWL}}$ & Storz $\mathrm{XC}_{\mathrm{cccc}}$ \\
\hline $\mathrm{NaCl}$ & 2.372 & 2.942 & 1.233 & 3.084 & 2.417 & 1.395 & 4.359 & 4.472 \\
\hline Sterile Water & 2.447 & 2.854 & 1.172 & 3.288 & 2.443 & 1.272 & 4.256 & 4.547 \\
\hline Contrast & 2.634 & 2.663 & 1.298 & 3.566 & 2.602 & 1.388 & 4.197 & 4.466 \\
\hline Betadine & 2.343 & 2.583 & 1.227 & 3.453 & 2.615 & 1.440 & 3.667 & 3.916 \\
\hline Total & 2.449 & 2.761 & 1.232 & 3.348 & 2.519 & 1.374 & 4.120 & 4.350 \\
\hline
\end{tabular}


Figure and Table Legends:
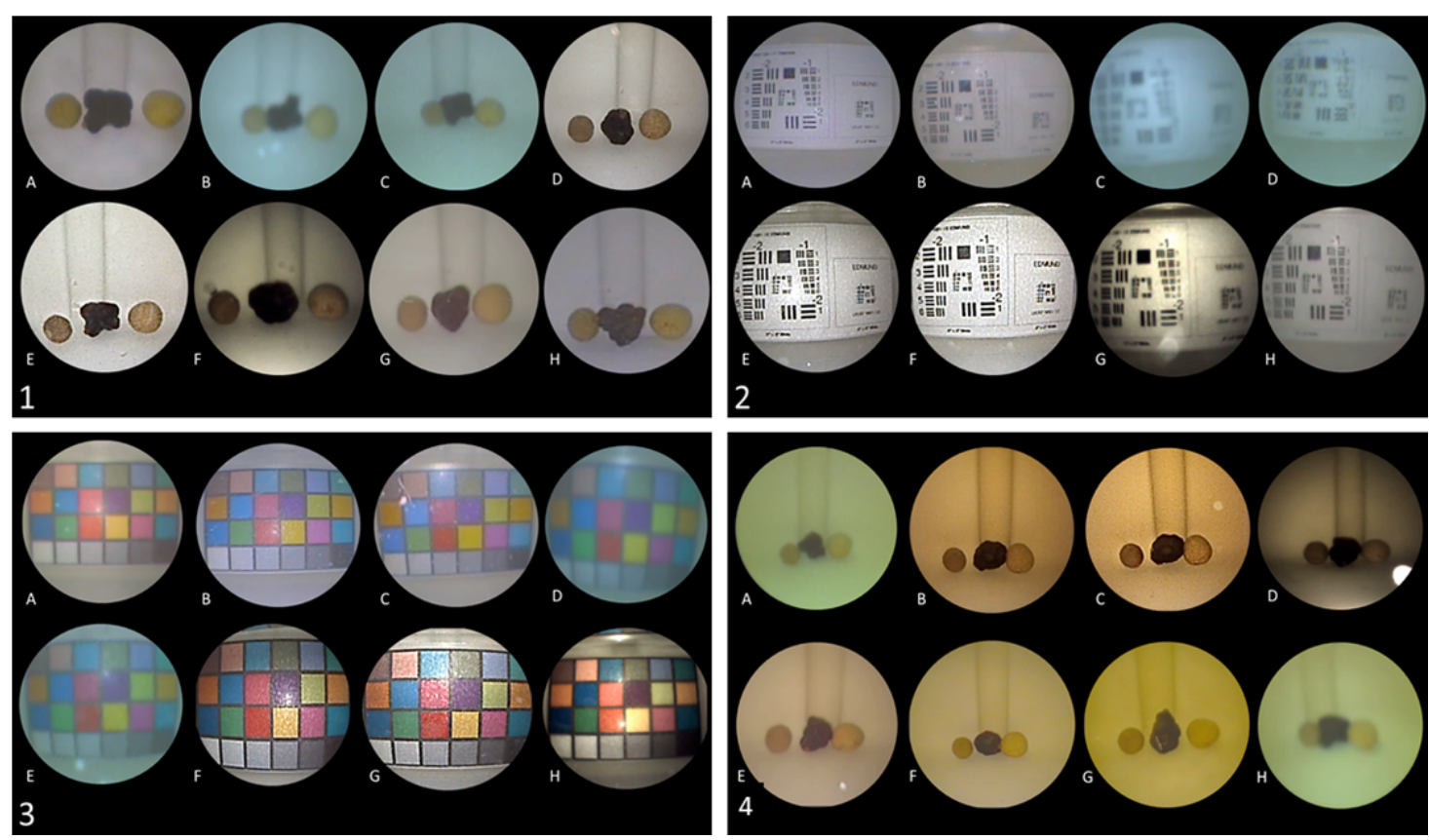

Figure 1. An example of four different templates. In each slide are eight videos of the same subject obtained with eight different FUs. 1) Stones in saline solution; 2) image definition in saline solution; 3 ) color contrast in saline solution; 4) stones in saline and Betadine solutions. In each slide, the position of the video of the FU was changed randomly. 
Page 12 of 16

12

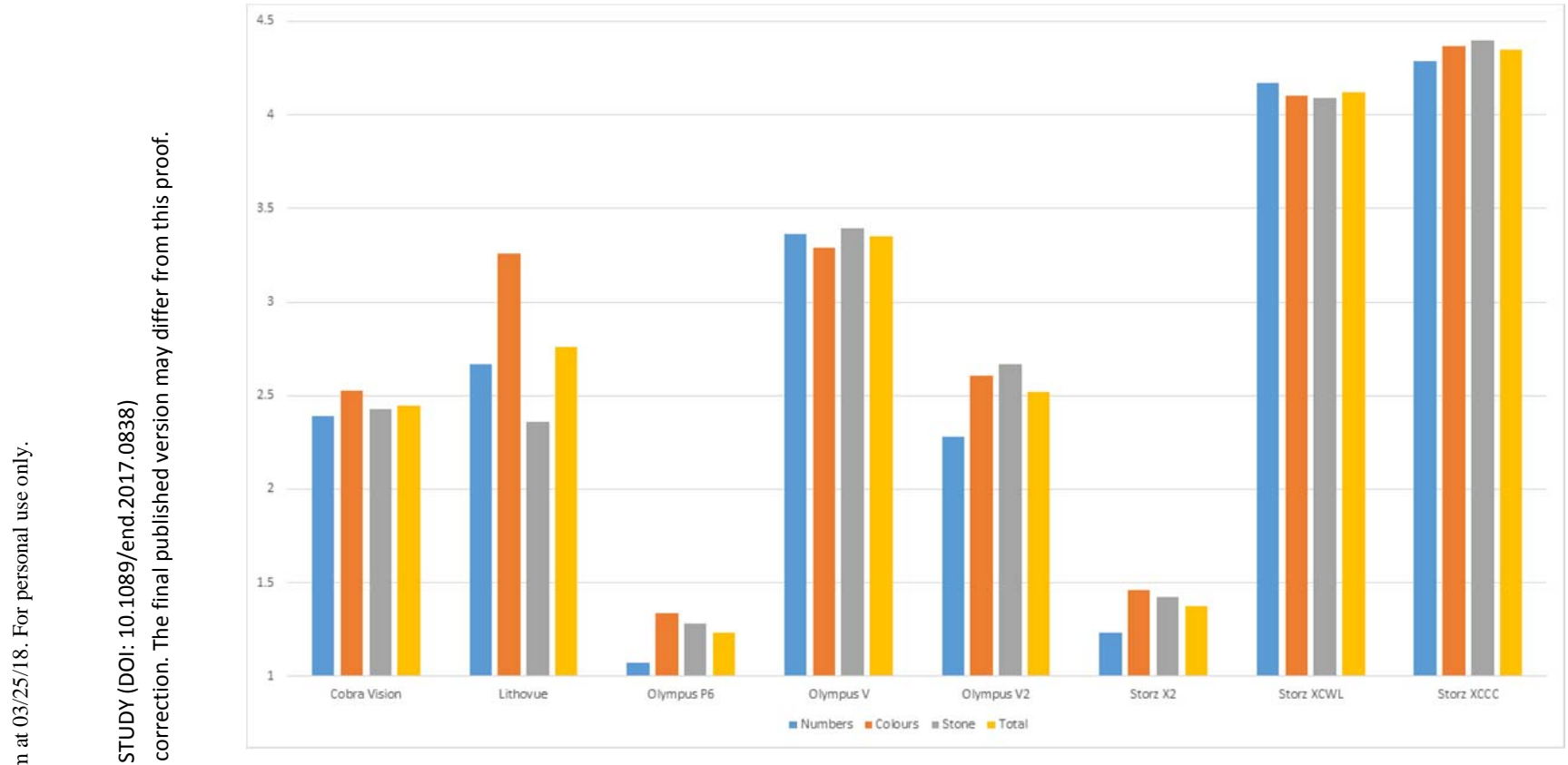

Figure 2. Histogram representing the mean evaluations of the FUs with different targets. 1

$=$ very bad, 5 = very good 


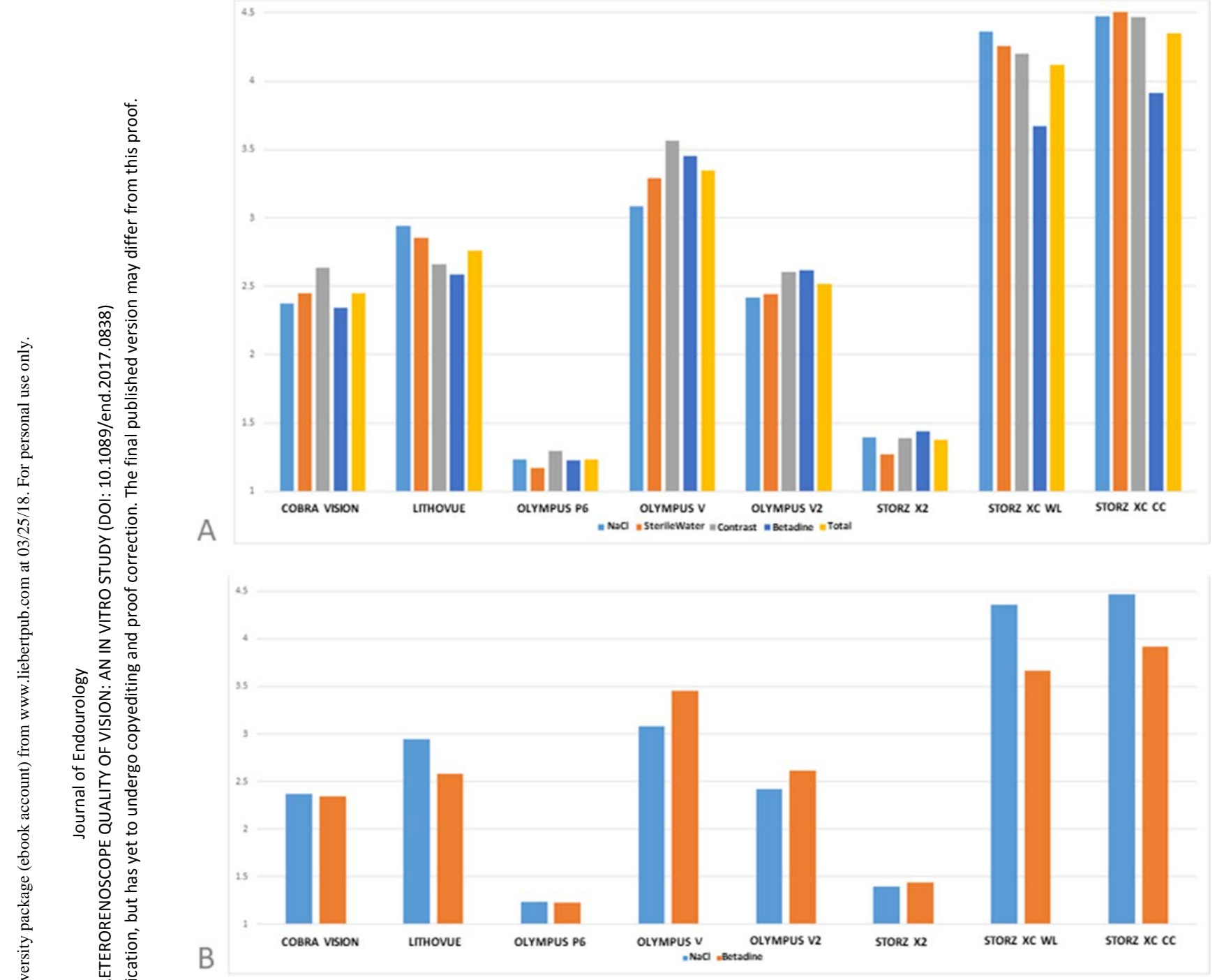

Figure 3. A) Histogram representing the mean evaluations of the FUs in different mediums. $1=$ very bad, 5 = very good. B) Comparison between views in saline solution and Betadine solution 
Page 14 of 16

14

Paris, /10/2015

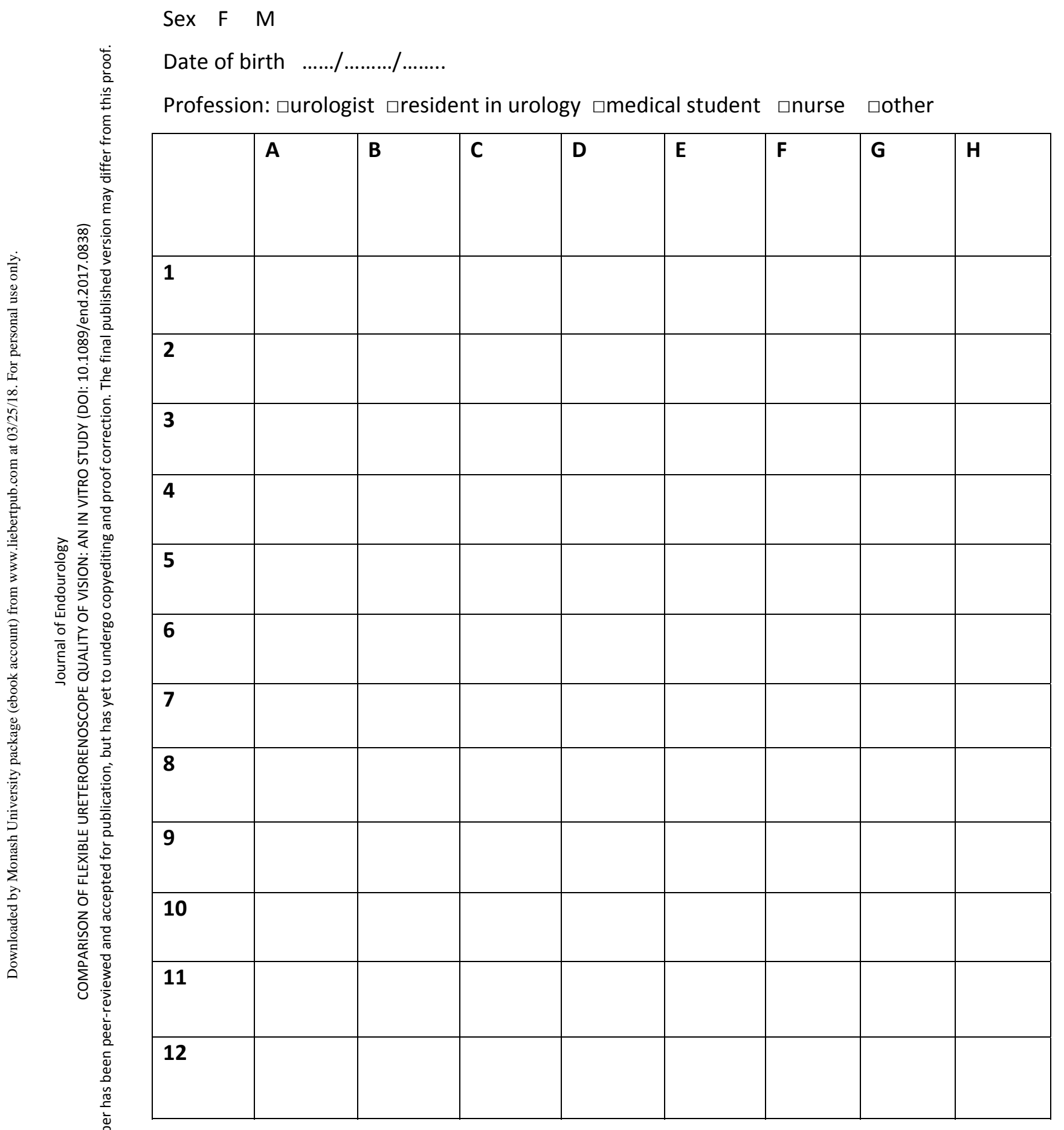

1= very bad; $2=$ bad; 3=normal; 4=good; 5=very good 


\section{References}

'EAU Guidelines 2017. Upper urinary tract urothelial carcionoma.

http://uroweb.org/guideline/upper-urinary-tract-urothelial-cell-carcinoma/

ii Quayle SS, Ames CD, Lieber D, Yan Y, Landman J. Comparison of optical resolution with digital and standard fiberoptic cystoscopes in an in vitro model. Urology 2015;66:489-493

iii Abdelshehid C, Ahlering MT, Chou D, Park HK, Basillote J, Lee D, et al. Comparison of flexible ureteroscopes: deflection, irrigant flow and optical characteristics. J Urol 2005;173:2017-2021

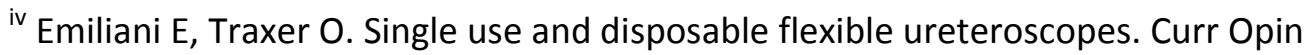
2017;27:176-181

${ }^{v}$ Proietti S, Dragos L, Molina W, Doizi S, Giusti G, Traxer O. Comparison of new single-use digital flexible ureteroscope versus nondisposable fiber optic and digital ureteroscope in a cadaveric model. J Endourol 2016;30:655-659

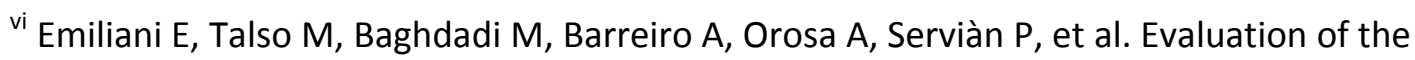
Spies $^{\mathrm{TM}}$ modalities image quality. Int Braz J Urol. 2017;24;43.

vii USAF 1951. From website of Edmund Optics, Barrington, NJ

viii Color Checker. From website of Edmund Optics, Barrington, NJ

${ }^{\text {ix }}$ Somani BK, Al-Qahtani SM, de Medina SD, Traxer O. Outcomes of flexible ureterorenoscopy and laser fragmentation for renal stones: comparison between digital and conventional ureteroscope. Urology 2013;82:1017-1019

× Mandalapu RS, Remzi M, de Reijke TM, Margulis V, Palou J, Kapoor A, et al. Update of the ICUD-SIU consultation on upper tract urothelial carcinoma 2016: treatment of low-risk upper tract urothelial carcinoma. World J Urol 2017;35:355-365.

${ }^{x i}$ Dale J, Kaplan AG, Radvak D, Shin R, Ackerman A, Chen T, et al. Evaluation of a novel single-use flexible ureteroscope. J Endourol. 2017 Mar 2. doi: 10.1089/end.2016.0237.

xii Talso M, Emiliani E, Baghdadi M, Orosa A, Servian $\mathrm{P}$, Barreiro A, et al. The new grasperintegrated single use flexible cystoscope for double J stent removal: evaluation of image quality, flow and flexibility. World J Urol. 2017;35:1277-1283.

xiii Karl Storz Flex XC brochure: accessed on 15 Mar 2017. Available at. www.karlstorz.com/cps/rde/xbcr/karlstorz_assets/ASSETS/3347684.pdf 
${ }^{\text {xiv }}$ Dragos LB, Somani BK, Sener ET, Buttice S, Proietti S, Ploumidis A, lacoboaie CT, Doizi

S, Traxer O Which Flexible Ureteroscopes (Digital vs. Fiber-Optic) Can Easily Reach the Difficult Lower Pole Calices and Have Better End-Tip Deflection: In Vitro Study on K-Box. A PETRA Evaluation. J Endourol. 2017 Jul;31(7):630-637. doi: 10.1089/end.2017.0109. Epub 2017 Jun 13.

${ }^{x v}$ Proietti S, Somani B, Sofer M, Pietropaolo A, Rosso M, Saitta G, Gaboardi F, Traxer O, Giusti G The "Body Mass Index" of Flexible Ureteroscopes. J Endourol. 2017 Oct;31(10):1090-1095. doi: 10.1089/end.2017.0438. Epub 2017 Sep 28 